\title{
Study of sardine oil antioxidant properties for the development of topical therapeutic formulations
}

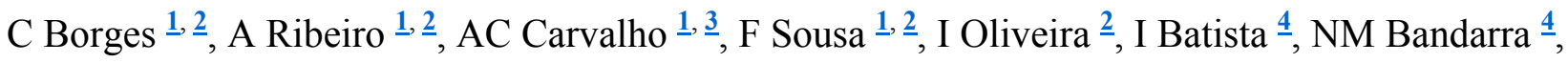
AC Gomes $\stackrel{2}{2}$, A Cavaco-Paulo $\stackrel{1}{-}$

- ${ }^{1} \mathrm{CEB}$ - Centre of Biological Engineering, Department of Biological Engineering, University of Minho, Campus of Gualtar, 4710 - 057 Braga, Portugal

- ${ }^{2}$ Centre of Molecular and Environmental Biology (CBMA), Department of Biology, University of Minho, Campus of Gualtar, 4710 - 057 Braga, Portugal

- ${ }^{3}$ CITAB - Centre for the Research and Technology of Agro-Environmental and Biological Sciences, Department of Biology, University of Minho, Campus of Gualtar, 4710 - 057 Braga, Portugal

- ${ }^{4}$ Instituto Português do Mar e da Atmosfera (IPMA), Av. de Brasília, 1449 - 006 Lisboa, Portugal

- $\underline{\text { Congress Abstract }}$

Sardine is one of the most common fish of the Portuguese coast with important nutritional features. Sardine oil is also a natural source of nutrients with proven benefits for human health, being rich in omega-3 fatty acids, particularly EPA and DHA (polyunsaturated fatty acids-PUFAs) [1]. Several studies show that there is a direct link between a diet enriched in omega-3 and the prevention of diseases such as cardiovascular disease, inflammatory conditions, mental disorders and prevention of various types of cancer [2]. The aim of this work was to characterize the antioxidant role of sardine oil for the development of topical applications. To evaluate the antioxidant effect of sardine oil on skin, human fibroblasts (BJ-5ta), human melanocytes (A375) and human keratinocytes (NCTC2544) were used. Concentrations of oil higher than $8 \mathrm{mg} / \mathrm{ml}$ affected significantly the cell viability while for lower concentrations the effect was reduced. The lowest concentrations, 0.5 and $4 \mathrm{mg} / \mathrm{ml}$, were tested to evaluate the protective role of sardine oil in situation of induced oxidative stress. These two concentrations were able to protect cells from damage with a higher effect measured for the fibroblasts. Moreover the incubation of cells with the sardine oil was able to activate a transcription factor - Nuclear factor-erythroid-2-related factor 2 (Nrf2) - which plays a crucial role in the coordinated induction of genes encoding many stressresponsive and cytoprotective enzymes and related proteins [3]. These results open the opportunity to develop new therapeutic and cosmetic applications based on sardine-derived compounds. Their incorporation in topical creams may contribute to a better treatment of inflammation and in the prevention of skin aging.

\section{References:}

[1] Calder P, Yaqoob P. Omega-3 polyunsaturated fatty acids and human health outcomes. Biofactors 2009; 65: $266-272$.

[2] Allayee H, Roth N, Hodis HN. Polyunsaturated fatty acids and cardiovascular disease: implications for nutrigenetics. J.Nutrigenet Nutrigenomics 2009; 2:140 - 148. 
[3] S YJ, Kundu KJ, Na HK. Nrf2 as a Master Redox Switch in Turning on the Cellular Signaling Involved in the Induction of Cytoprotective Genes by Some Chemopreventive Phytochemicals. Planta Med 2009; 74:1526 - 1539. 